Math. Proc. Camb. Phil. Soc. (2021), 171, 51-64 C Cambridge Philosophical Society 2020. This is an Open Access article, distributed under the terms of the Creative Commons Attribution-NonCommercial-ShareAlike licence (http://creativecommons.org/licenses/by-nc-sa/4.0/), which permits non-commercial re-use, distribution, and reproduction in any medium, provided the same Creative Commons licence is included and the original work is properly cited. The written permission of Cambridge University Press must be obtained for commercial re-use. doi:10.1017/S0305004120000055

First published online 16 March 2020

\title{
Two polarised K3 surfaces associated to the same cubic fourfold
}

\author{
BY EMMA BRAKKEE \\ Korteweg-de Vries Institute, University of Amsterdam, \\ P.O. Box 94248, 1090 GE Amsterdam, Netherlands. \\ e-mail:e.1.brakkee@uva.n1
}

(Received 05 December 2018; revised 11 September 2019)

\begin{abstract}
For infinitely many $d$, Hassett showed that special cubic fourfolds of discriminant $d$ are related to polarised K3 surfaces of degree $d$ via their Hodge structures. For half of the $d$, each associated K3 surface $(S, L)$ canonically yields another one, $\left(S^{\tau}, L^{\tau}\right)$. We prove that $S^{\tau}$ is isomorphic to the moduli space of stable coherent sheaves on $S$ with Mukai vector $(3, L, d / 6)$. We also explain for which $d$ the Hilbert schemes $\operatorname{Hilb}^{n}(S)$ and $\operatorname{Hilb}^{n}\left(S^{\tau}\right)$ are birational.
\end{abstract}

2010 Mathematics Subject Classification: 14J28 (primary), 14J10, 14E05 (secondary)

Special cubic fourfolds were first studied by Hassett [9]. They are distinguished by the property that they carry additional algebraic cycles. They arise in countably many families, parametrised by irreducible divisors $\mathcal{C}_{d}$ in the moduli space of cubic fourfolds. For infinitely many $d$, the cubic fourfolds in $\mathcal{C}_{d}$ are related to polarised K3 surfaces of degree $d$ via their Hodge structures. For half of the $d, \mathrm{~K} 3$ surfaces associated to generic cubics in $\mathcal{C}_{d}$ come in pairs. The goal of this paper is to explain how two such $\mathrm{K} 3$ surfaces are related.

More precisely, denote by $\mathcal{M}_{d}$ the moduli space of polarised K3 surfaces of degree $d$. Hassett constructed, for admissible $d$, a surjective rational map $\mathcal{M}_{d}-\rightarrow \mathcal{C}_{d}$ sending a K3 surface to a cubic fourfold it is associated to. This map is of degree two when $d \equiv 0$ mod 6 and generically injective otherwise. In the former case, its (regular) covering involution $\tau: \mathcal{M}_{d} \rightarrow \mathcal{M}_{d}$ does not depend on the choices made to construct $\mathcal{M}_{d}-\rightarrow \mathcal{C}_{d}$. We prove the following geometric description of $\tau$.

TheOREM 1 (see Theorem 3.2). Let $\left(S^{\tau}, L^{\tau}\right)=\tau(S, L)$. Then $S^{\tau}$ is isomorphic to the moduli space $M_{S}(v)$ of stable (with respect to a generic polarisation) coherent sheaves on $S$ with Mukai vector $v=(3, L, d / 6)$.

In particular, $S$ and $S^{\tau}$ are Fourier-Mukai partners. For generic $(S, L) \in \mathcal{M}_{d}$, this also follows from the fact that the bounded derived categories of $S$ and $S^{\tau}$ are both exact equivalent to the Kuznetsov category of the image cubic fourfold [2]. If $\rho(S)=1$, then $S$ is not isomorphic to $S^{\tau}$ (as unpolarised K3 surfaces). The number of Fourier-Mukai partners of $S$, which depends on $d$, can be arbitrarily high [17]. The above gives a natural way of selecting one of them for each $(S, L) \in \mathcal{M}_{d}$. 
We also explain when the Hilbert schemes of $n$ points $\operatorname{Hilb}^{n}(S)$ and $\operatorname{Hilb}^{n}\left(S^{\tau}\right)$ are birational. Our main result is the following.

TheOREM 2 (see Proposition 4.5, Corollary 4.9). Let $d \equiv 0$ mod 6 satisfy (**). Consider the following statements:

(i) $\operatorname{Hilb}^{2}(S)$ is isomorphic to $\operatorname{Hilb}^{2}\left(S^{\tau}\right)$;

(ii) $\operatorname{Hilb}^{2}(S)$ is birational to $\operatorname{Hilb}^{2}\left(S^{\tau}\right)$;

(iii) there exists an integral solution to $3 p^{2}-(d / 6) q^{2}=-1$;

(iv) $\operatorname{Hilb}^{2}(S)$ has a line bundle of self-intersection 6.

We have implications (i) $\Rightarrow$ (ii) $\Leftarrow$ (iii) $\Rightarrow$ (iv). If $\rho(S)=1$, then these are all equivalent.

We will see that the condition in (iii) is satisfied for infinitely many $d$ but not for all of them. As an application, we obtain an example of derived equivalent Hilbert schemes of two points on $\mathrm{K} 3$ surfaces which are not birational.

\section{Lattices}

In this section we set up the notation for the lattice theory that will be needed. See [12, Section 2] for references.

For a lattice $\Lambda$ with intersection form (, ) : $\Lambda \times \Lambda \rightarrow \mathbb{Z}$, we denote by $\Lambda^{\vee}$ its dual lattice and by Disc $\Lambda=\Lambda^{\vee} / \Lambda$ its discriminant group. Every orthogonal transformation $g \in \mathrm{O}(\Lambda)$ of $\Lambda$ induces an automorphism on Disc $\Lambda$, which we denote by $\bar{g}$. When $L$ is even, the product $($, ) induces a quadratic form

$$
q_{L}: \operatorname{Disc} L \longrightarrow \mathbb{Q} / 2 \mathbb{Z} \text {. }
$$

We denote by $\mathrm{O}$ (Disc $L$ ) the group of automorphisms preserving $q_{L}$. We further define

$$
\widetilde{\mathrm{O}}(L):=\operatorname{ker}(\mathrm{O}(L) \longrightarrow \mathrm{O}(\operatorname{Disc} L)) .
$$

We will use a result that is slightly stronger than [11, Proposition 14.2.6], but is proven in the same way. We give the proof here for completeness.

Let $\Lambda_{1}$ be a primitive sublattice of a unimodular lattice $\Lambda$ and let $\Lambda_{2} \subset \Lambda$ be its orthogonal complement. We have an inclusion $\Lambda^{\vee} \subset\left(\Lambda_{1} \oplus \Lambda_{2}\right)^{\vee}$ which induces

$$
\Lambda^{\vee} /\left(\Lambda_{1} \oplus \Lambda_{2}\right) \longleftrightarrow \operatorname{Disc}\left(\Lambda_{1} \oplus \Lambda_{2}\right) \cong \operatorname{Disc}\left(\Lambda_{1}\right) \oplus \operatorname{Disc}\left(\Lambda_{2}\right) .
$$

The projection maps $\Lambda^{\vee} /\left(\Lambda_{1} \oplus \Lambda_{2}\right) \rightarrow \operatorname{Disc}\left(\Lambda_{i}\right)$ are isomorphisms, by unimodularity of $\Lambda$ and primitivity of $\Lambda_{1}$ and $\Lambda_{2}$. This gives an isomorphism $\varphi: \operatorname{Disc}\left(\Lambda_{1}\right) \rightarrow \operatorname{Disc}\left(\Lambda_{2}\right)$ sending $\bar{x} \in \operatorname{Disc}\left(\Lambda_{1}\right)$ to the unique class $\bar{y} \in \operatorname{Disc}\left(\Lambda_{2}\right)$ such that $x+y \in \Lambda_{1}^{\vee} \oplus \Lambda_{2}^{\vee}$ is in $\Lambda$.

Lemma 1.1. If $g_{1} \in \mathrm{O}\left(\Lambda_{1}\right)$ and $g_{2} \in \mathrm{O}\left(\Lambda_{2}\right)$, then $g_{1} \oplus g_{2}: \Lambda_{1} \oplus \Lambda_{2} \rightarrow \Lambda_{1} \oplus \Lambda_{2}$ extends to an automorphism of $\Lambda$ if and only if $\bar{g}_{1}=\bar{g}_{2}$ under the identification Disc $\Lambda_{1} \cong$ Disc $\Lambda_{2}$.

Proof. The map $g_{1} \oplus g_{2}$ extends to $L$ if and only if for all $x_{1} \in \Lambda_{1}^{\vee}, x_{2} \in \Lambda_{2}^{\vee}$ with $x_{1}+x_{2} \in \Lambda$, the element $g_{1}^{\vee}\left(x_{1}\right)+g_{2}^{\vee}\left(x_{2}\right)$ also lies in $\Lambda$. We have $x_{1}+x_{2} \in \Lambda$ if and only if $\varphi\left(\bar{x}_{1}\right)=\bar{x}_{2}$. So $g_{1}^{\vee}\left(x_{1}\right)+g_{2}^{\vee}\left(x_{2}\right)$ is in $\Lambda$ if and only if $\varphi\left(\bar{g}_{1}\left(\bar{x}_{1}\right)\right)$ equals $\bar{g}_{2}\left(\bar{x}_{2}\right)=\bar{g}_{2}\left(\varphi\left(\bar{x}_{1}\right)\right)$. This holds for all $x_{1}, x_{2}$ if and only if $\varphi \circ \bar{g}_{1}=\bar{g}_{2} \circ \varphi$.

The middle cohomology $\mathrm{H}^{2}(S, \mathbb{Z})$ of a $\mathrm{K} 3$ surface $S$ (with the usual intersection pairing) is isomorphic to the $\mathrm{K} 3$ lattice 


$$
\Lambda_{\mathrm{K} 3}:=E_{8}(-1)^{\oplus 2} \oplus U^{\oplus 3}=E_{8}(-1)^{\oplus 2} \oplus U_{1} \oplus U_{2} \oplus U_{3} .
$$

We denote the standard basis of $U_{i}$ by $e_{i}, f_{i}$. On the full cohomology $\mathrm{H}^{*}(S, \mathbb{Z})$ of $S$ we consider the Mukai pairing, given by $\left(\left(x_{0}, x_{2}, x_{4}\right),\left(x_{0}^{\prime}, x_{2}^{\prime}, x_{4}^{\prime}\right)\right)=x_{2} x_{2}^{\prime}-x_{0} x_{4}^{\prime}-x_{0}^{\prime} x_{4}$ for $x_{i}, x_{i}^{\prime} \in \mathrm{H}^{i}(S, \mathbb{Z})$. With this pairing, $\mathrm{H}^{*}(S, \mathbb{Z})$ becomes isomorphic to the Mukai lattice

$$
\Lambda_{\mathrm{Muk}}:=\Lambda_{\mathrm{K} 3} \oplus U(-1)=E_{8}(-1)^{\oplus 2} \oplus U_{1} \oplus U_{2} \oplus U_{3} \oplus U_{4}(-1) .
$$

As $U \cong U(-1)$, the Mukai lattice is isomorphic to $\Lambda_{\mathrm{K} 3} \oplus U$. To avoid confusion, we denote the latter by $\widetilde{\Lambda}_{\mathrm{K} 3}$, and fix an isomorphism $\widetilde{\Lambda}_{\mathrm{K} 3} \stackrel{\sim}{\rightarrow} \Lambda_{\mathrm{Muk}}$ by sending $f_{4}$ to $-f_{4}$.

Fix $\ell_{d}=e_{3}+(d / 2) f_{3} \in U_{3} \subset \Lambda_{\mathrm{K} 3}$ and let $\Lambda_{d}:=\ell_{d}^{\perp} \subset \Lambda_{\mathrm{K} 3}$ be its orthogonal complement in $\Lambda_{\mathrm{K} 3}$. Then

$$
\Lambda_{d}=E_{8}(-1)^{\oplus 2} \oplus U^{\oplus 2} \oplus \mathbb{Z}(-d)
$$

is isomorphic to the primitive cohomology $L^{\perp} \subset \mathrm{H}^{2}(S, \mathbb{Z})$ of any polarised $\mathrm{K} 3$ surface $(S, L)$ of degree $d$.

We denote by $\mathrm{H}^{4}(X, \mathbb{Z})(1)$ the middle cohomology of a cubic fourfold $X$, with the weight of the Hodge structure shifted by two and the intersection product changed by a sign. As a lattice, it is isomorphic to

$$
\Lambda_{\text {cub }}:=E_{8}(-1)^{\oplus 2} \oplus U^{\oplus 2} \oplus \mathbb{Z}(-1)^{\oplus 3} .
$$

Let $h=(1,1,1) \in \mathbb{Z}(-1)^{\oplus 3} \subset \Lambda_{\text {cub. }}$. The primitive cohomology $\mathrm{H}^{4}(X, \mathbb{Z})_{\text {prim }}(1)$ of $X$ is isomorphic to $\Lambda_{\text {cub }}^{0}:=h^{\perp} \subset \Lambda_{\text {cub }}$. An easy computation shows that

$$
\Lambda_{\text {cub }}^{0} \cong E_{8}(-1)^{\oplus 2} \oplus U^{\oplus 2} \oplus A_{2}(-1),
$$

where $A_{2}$ is the lattice $\left(\mathbb{Z}^{\oplus 2},\left(\begin{array}{cc}2 & -1 \\ -1 & 2\end{array}\right)\right)$. There exists a primitive embedding of $A_{2}$ into $U_{3} \oplus$ $U_{4} \subset \widetilde{\Lambda}_{\mathrm{K} 3}$, unique up the action of $\mathrm{O}\left(\widetilde{\Lambda}_{\mathrm{K} 3}\right)$. One can show that $\Lambda_{\text {cub }}^{0} \cong A_{2}^{\perp} \subset \widetilde{\Lambda}_{\mathrm{K} 3}$.

\section{Hassett's construction}

We summarise Hassett's construction, explaining those proofs that we need for our results. For details, see [9].

\subsection{Special cubic fourfolds}

Inside $\mathrm{H}^{4}(X, \mathbb{Z})(1)$, we consider the negative definite lattice

$$
A(X):=\mathrm{H}^{4}(X, \mathbb{Z})(1) \cap \mathrm{H}^{2,2}(X) .
$$

We fix the notation $h_{X}^{2} \in \mathrm{H}^{4}(X, \mathbb{Z})$ for the square of the hyperplane class on $X$. For $X$ very general, the lattice $A(X)$ has rank one and is generated by $h_{X}^{2}$. We call $X$ special if rk $A(X) \geq$ 2. By the Hodge conjecture for cubic fourfolds [22], $X$ is special if and only if $X$ contains a surface that is not homologous to a complete intersection.

If $X$ is special, then $A(X)$ contains a primitive sublattice $K$ of rank two. Hassett proved that fixing the discriminant disc $K$ of such $K$ gives divisors in the moduli space $\mathcal{C}$ of smooth cubic fourfolds. Namely, define

$$
\mathcal{C}_{d}:=\left\{X \in \mathcal{C} \mid \exists K \subset A(X) \text { primitive, } h_{X}^{2} \in K \text {, rk } K=2 \text {, disc } K=d\right\} .
$$

Then the set of special cubic fourfolds in $\mathcal{C}$ is the union of all $\mathcal{C}_{d}$. 
THEOREM $2 \cdot 1\left(\left[9\right.\right.$, Theorem 1·0.1]). The set $\mathcal{C}_{d}$ is either empty or an irreducible divisor in $\mathcal{C}$. It is non-empty if and only if $d$ satisfies

$$
d>6 \text { and } d \equiv 0,2 \bmod 6 .
$$

\subsection{Periods of special cubic fourfolds}

Recall the definition of the period domain for a lattice $\Lambda$ of signature $\left(n_{+}, n_{-}\right)$with $n_{+} \geq 2$ :

$$
\mathcal{D}(\Lambda)=\left\{x \in \mathbb{P}(\Lambda \otimes \mathbb{C}) \mid(x)^{2}=0,(x, \bar{x})>0\right\} .
$$

This is a complex manifold of dimension rk $\Lambda-2$, which is connected when $n_{+}>2$ and has two connected components when $n_{+}=2$. It has a natural action by the group $\widetilde{\mathrm{O}}(\Lambda)$. This is an arithmetic group; if $n_{+}=2$, then by [20, Section 6 of appendix] and [4], the quotient

$$
\mathcal{Q D}(\Lambda):=\mathcal{D}(\Lambda) / \widetilde{\mathrm{O}}(\Lambda)
$$

is a (connected) quasi-projective variety with at most finite quotient singularities.

In particular, this holds for $\Lambda=\Lambda_{d}$, yielding a 19-dimensional variety $\mathcal{Q D}\left(\Lambda_{d}\right)$. The period map induces an open embedding $\mathcal{M}_{d} \hookrightarrow \mathcal{Q D}\left(\Lambda_{d}\right)$ (see e.g. [11, Corollary 6.4.3]).

The lattice $\Lambda_{\text {cub }}^{0}$ also satisfies $n_{+}=2$; the variety $\mathcal{Q D}\left(\Lambda_{\text {cub }}^{0}\right)$ has dimension 20 . The period map gives an open embedding $\mathcal{C} \hookrightarrow \mathcal{Q D}\left(\Lambda_{\text {cub }}^{0}\right)[\mathbf{9}$, Section 2.2].

Inside $\mathcal{D}\left(\Lambda_{\text {cub }}^{0}\right)$, we can identify those periods coming from special cubic fourfolds. Note that a cubic fourfold $X$ is special if and only if there exists a negative definite sublattice $K \subset \mathrm{H}^{4}(X, \mathbb{Z})(1)$ of rank two with $h_{X}^{2} \in K$, such that $K \otimes \mathbb{C}$ is contained in $\mathrm{H}^{3,1}(X)^{\perp} \subset \mathrm{H}^{4}(X, \mathbb{C})(1)$. On the level of the period domain, this means the following: After choosing a marking $\mathrm{H}^{4}(X, \mathbb{Z})_{\text {prim }}(1) \stackrel{\sim}{\rightarrow} \Lambda_{\text {cub }}^{0}$, the period of $X$ lands in

$$
\left\{x \in \mathcal{D}\left(\Lambda_{\text {cub }}^{0}\right) \mid\left(K \cap \Lambda_{\text {cub }}^{0}\right) \mathbb{C} \subset x^{\perp}\right\}
$$

for some primitive, negative definite sublattice $K \subset \Lambda_{\text {cub }}$ of rank two containing $h$. Let $K^{\perp}$ be its orthogonal complement; note that $K^{\perp} \subset \Lambda_{\text {cub }}^{0}$. The set above is the divisor $\mathcal{D}\left(K^{\perp}\right) \subset \mathcal{D}\left(\Lambda_{\text {cub }}^{0}\right)$.

We fix a primitive sublattice $K_{d} \subset \Lambda_{\text {cub }}$ as above, of discriminant $d$. Let $\overline{\mathcal{C}}_{d} \subset \mathcal{Q D}\left(\Lambda_{\text {cub }}^{0}\right)$ be the image of $\mathcal{D}\left(K_{d}^{\perp}\right) \subset \mathcal{D}\left(\Lambda_{\text {cub }}^{0}\right)$ under the quotient map $\mathcal{D}\left(\Lambda_{\text {cub }}^{0}\right) \rightarrow \mathcal{Q D}\left(\Lambda_{\text {cub }}^{0}\right)$. The following shows that $\overline{\mathcal{C}}_{d}$ does not depend on the choice of $K_{d}$.

Proposition $2 \cdot 2$ ([9, Proposition 3.2.4]). Let $K, K^{\prime} \subset \Lambda_{\text {cub }}$ be primitive sublattices of rank two containing $h$. Then $K=f\left(K^{\prime}\right)$ for some $f \in \widetilde{\mathrm{O}}\left(\Lambda_{\mathrm{cub}}^{0}\right)$ if and only if disc $K=$ $\operatorname{disc} K^{\prime}$.

Note that the immersion $\mathcal{C} \hookrightarrow \mathcal{Q D}\left(\Lambda_{\text {cub }}^{0}\right)$ maps $\mathcal{C}_{d}$ into $\overline{\mathcal{C}}_{d}$. In fact, we have $\mathcal{C}_{d}=\mathcal{C} \cap \overline{\mathcal{C}}_{d}$.

\subsection{Associated $K 3$ surfaces}

Consider the following condition on $d \in \mathbb{N}$ :

$$
d \text { is even and not divisible by } 4,9 \text {, or any odd prime } p \equiv 2 \bmod 3 .
$$

This implies that $d \equiv 0,2 \bmod 6$. Hassett proved the following statement: 
Proposition 2.3 ([9, Proposition 5.1.4]). The number $d$ satisfies (**) if and only if there is an isomorphism $\Lambda_{d} \cong K_{d}^{\perp}$.

So when $d$ satisfies $(* *)$, there is an isomorphism of period domains $\mathcal{D}\left(\Lambda_{d}\right) \cong \mathcal{D}\left(K_{d}^{\perp}\right)$. Proposition 2.6 below tells us that under the identification $\Lambda_{d} \cong K_{d}^{\perp}$, the group $\widetilde{\mathrm{O}}\left(\Lambda_{d}\right)$ forms a subgroup of $\widetilde{\mathrm{O}}\left(\Lambda_{\text {cub }}^{0}\right)$. Hence, we also get a map from $\mathcal{Q D}\left(\Lambda_{d}\right)=\mathrm{O}\left(\Lambda_{d}\right) \backslash \mathcal{D}\left(\Lambda_{d}\right)$ to $\overline{\mathcal{C}}_{d}$. This gives us the following commutative diagram:

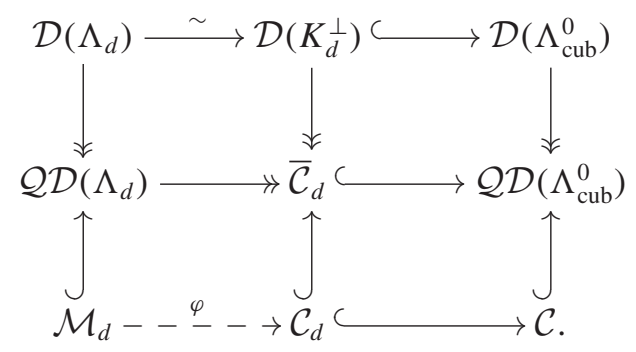

It can be shown that the rational map $\varphi: \mathcal{M}_{d}-\rightarrow \mathcal{C}_{d}$ is regular on an open subset which maps surjectively to $\mathcal{C}_{d}$, see [9, Section 5.1]. Note that $\varphi$ depends on the choice of an isomorphism $\Lambda_{d} \cong K_{d}^{\perp}$, thus it is only unique up to $\mathrm{O}\left(\Lambda_{d}\right) / \widetilde{\mathrm{O}}\left(\Lambda_{d}\right)$.

If $\varphi$ sends $(S, L) \in \mathcal{M}_{d}$ to $X$, then there exists an isometry of Hodge structures

$$
\mathrm{H}^{4}(X, \mathbb{Z})(1) \supset K^{\perp} \cong L^{\perp} \subset \mathrm{H}^{2}(S, \mathbb{Z})
$$

for some primitive sublattice $K \subset A(X)$ of rank two and discriminant $d$ containing $h_{X}^{2}$. Conversely, if such a Hodge isometry exists, it induces a lattice isomorphism

$$
\Lambda_{d} \cong L^{\perp} \cong K^{\perp} \cong K_{d}^{\perp}
$$

such that the induced map $\varphi: \mathcal{M}_{d}-\rightarrow \mathcal{C}_{d}$ sends $(S, L)$ to $X$. This motivates the following definition.

Definition 2.4. Let $X \in \mathcal{C}_{d}$. A polarised K3 surface $(S, L) \in \mathcal{M}_{d}$ is associated to $X$ if there exists a Hodge isometry

$$
\mathrm{H}^{4}(X, \mathbb{Z})(1) \supset K^{\perp} \cong L^{\perp} \subset \mathrm{H}^{2}(S, \mathbb{Z})
$$

for some primitive sublattice $K \subset A(X)$ of rank two and discriminant $d$ containing $h_{X}^{2}$.

For the rest of this section, we fix one choice of the rational map $\varphi$.

Remark 2.5. The complement of the image of the inclusion $\mathcal{C} \hookrightarrow \mathcal{Q D}\left(\Lambda_{\text {cub }}^{0}\right)$ is $\overline{\mathcal{C}}_{2} \cup \overline{\mathcal{C}}_{6}[\underline{13}$, 14]. Therefore, $\varphi(S, L)$ is defined if and only if the image of $(S, L)$ under $\mathcal{Q D}\left(\Lambda_{d}\right) \rightarrow \overline{\mathcal{C}}_{d}$ lies in $\overline{\mathcal{C}}_{d} \backslash\left(\overline{\mathcal{C}}_{2} \cup \overline{\mathcal{C}}_{6}\right)$. In particular, this holds when $\rho(S)=1$.

To describe the map $\mathcal{Q D}\left(\Lambda_{d}\right) \rightarrow \overline{\mathcal{C}}_{d}$, we define two subgroups of $\widetilde{\mathrm{O}}\left(\Lambda_{\text {cub }}^{0}\right)$. Let $v_{d}$ be a generator of $K_{d} \cap \Lambda_{\text {cub }}^{0}$, which is unique up to a sign. Define $\widetilde{\mathrm{O}}\left(\Lambda_{\text {cub }}^{0}, v_{d}\right) \subset \widetilde{\mathrm{O}}\left(\Lambda_{\text {cub }}^{0}\right)$ by

$$
\begin{aligned}
\widetilde{\mathrm{O}}\left(\Lambda_{\mathrm{cub}}^{0}, v_{d}\right) & :=\left\{f \in \mathrm{O}\left(\Lambda_{\mathrm{cub}}\right)|f|_{K_{d}}=\mathrm{id}_{K_{d}}\right\} \\
& =\left\{f \in \widetilde{\mathrm{O}}\left(\Lambda_{\mathrm{cub}}^{0}\right) \mid f\left(v_{d}\right)=v_{d}\right\} .
\end{aligned}
$$


The next statement is part of [9, Theorem 5.2.2]. It follows directly from Lemma 1·1.

Proposition 2.6. Suppose that $d$ satisfies (**). Under the isomorphism $\Lambda_{d} \cong K_{d}^{\perp}$, the group $\widetilde{\mathrm{O}}\left(\Lambda_{d}\right)$ is identified with $\widetilde{\mathrm{O}}\left(\Lambda_{\text {cub }}^{0}, v_{d}\right)$.

In particular, there is an isomorphism $\mathcal{Q D}\left(\Lambda_{d}\right) \cong \widetilde{\mathrm{O}}\left(\Lambda_{\text {cub }}^{0}, v_{d}\right) \backslash \mathcal{D}\left(K_{d}^{\perp}\right)$.

The second group we consider is

$$
\begin{aligned}
\widetilde{\mathrm{O}}\left(\Lambda_{\mathrm{cub}}^{0},\left\langle v_{d}\right\rangle\right) & =\left\{f \in \mathrm{O}\left(\Lambda_{\mathrm{cub}}\right) \mid f(h)=h \text { and } f\left(K_{d}\right)=K_{d}\right\} \\
& =\left\{f \in \widetilde{\mathrm{O}}\left(\Lambda_{\mathrm{cub}}^{0}\right) \mid f\left(v_{d}\right)= \pm v_{d}\right\} .
\end{aligned}
$$

By the Baily-Borel theorem [4], the quotient $\widetilde{\mathrm{O}}\left(\Lambda_{\text {cub }}^{0},\left\langle v_{d}\right\rangle\right) \backslash \mathcal{D}\left(K_{d}^{\perp}\right)$ is a normal quasiprojective variety. In fact, $\widetilde{\mathrm{O}}\left(\Lambda_{\text {cub }}^{0},\left\langle v_{d}\right\rangle\right) \backslash \mathcal{D}\left(K_{d}^{\perp}\right) \rightarrow \overline{\mathcal{C}}_{d}$ is the normalisation of $\overline{\mathcal{C}}_{d}$. Namely, a very general special cubic fourfold $X$ satisfies $\operatorname{rk} A(X)=2$ [21, Section 5.1], so there is only one sublattice $K_{d} \subset A(X)$. It follows that the map $\widetilde{\mathrm{O}}\left(\Lambda_{\text {cub }}^{0},\left\langle v_{d}\right\rangle\right) \backslash \mathcal{D}\left(K_{d}^{\perp}\right) \rightarrow \overline{\mathcal{C}}_{d}$ is generically injective. To see that it is proper, note that the action of $\widetilde{\mathrm{O}}\left(\Lambda_{\text {cub }}^{0}\right)$ on $\mathcal{D}\left(\Lambda_{\text {cub }}^{0}\right)$ is properly discontinuous [11, Remark 6.1.10]. Hence the map $\mathcal{D}\left(\Lambda_{\text {cub }}^{0}\right) \rightarrow \mathcal{Q D}\left(\Lambda_{\text {cub }}^{0}\right)$ is closed, as is its restriction $\mathcal{D}\left(K_{d}^{\perp}\right) \rightarrow \overline{\mathcal{C}}_{d}$ to the closed subset $\mathcal{D}\left(K_{d}^{\perp}\right) \subset \mathcal{D}\left(\Lambda_{\text {cub }}^{0}\right)$. Since this factors as

$$
\mathcal{D}\left(K_{d}^{\perp}\right) \rightarrow \widetilde{\mathrm{O}}\left(\Lambda_{\text {cub }}^{0},\left\langle v_{d}\right\rangle\right) \backslash \mathcal{D}\left(K_{d}^{\perp}\right) \rightarrow \overline{\mathcal{C}}_{d},
$$

the map $\widetilde{\mathrm{O}}\left(\Lambda_{\text {cub }}^{0},\left\langle v_{d}\right\rangle\right) \backslash \mathcal{D}\left(K_{d}^{\perp}\right) \rightarrow \overline{\mathcal{C}}_{d}$ is closed as well. Moreover, it has finite fibres, so it is proper.

Summarising, we have the following commutative diagram:

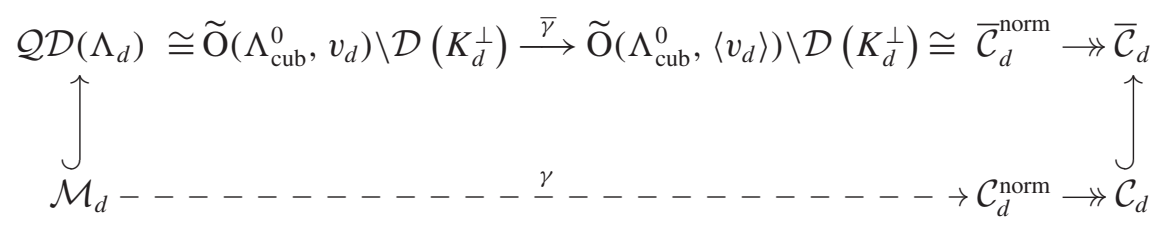

The spaces $\widetilde{\mathrm{O}}\left(\Lambda_{\text {cub }}^{0}, v_{d}\right) \backslash \mathcal{D}\left(K_{d}^{\perp}\right)$ and $\widetilde{\mathrm{O}}\left(\Lambda_{\text {cub }}^{0},\left\langle v_{d}\right\rangle\right) \backslash \mathcal{D}\left(K_{d}^{\perp}\right)$ can be seen as period domains of marked and labelled cubic fourfolds, respectively [9, Section 3.1, 5.2].

The following describes the generic fibre of the quotient map

$$
\bar{\gamma}: \widetilde{\mathrm{O}}\left(\Lambda_{\mathrm{cub}}^{0}, v_{d}\right) \backslash \mathcal{D}\left(K_{d}^{\perp}\right) \longrightarrow \widetilde{\mathrm{O}}\left(\Lambda_{\mathrm{cub}}^{0},\left\langle v_{d}\right\rangle\right) \backslash \mathcal{D}\left(K_{d}^{\perp}\right) .
$$

Proposition 2.7 ([9, proposition 5.2.1]). There is an isomorphism

$$
\widetilde{\mathrm{O}}\left(\Lambda_{\mathrm{cub}}^{0},\left\langle v_{d}\right\rangle\right) / \widetilde{\mathrm{O}}\left(\Lambda_{\mathrm{cub}}^{0}, v_{d}\right) \cong \begin{cases}\{0\} & \text { if } d \equiv 2 \bmod 6 \\ \mathbb{Z} / 2 \mathbb{Z} & \text { if } d \equiv 0 \bmod 6 .\end{cases}
$$

As a consequence, $\bar{\gamma}$ is an isomorphism when $d \equiv 2 \bmod 6$ and has degree two when $d \equiv 0 \bmod 6$. In the latter case, the covering involution of $\bar{\gamma}$ is induced by an automorphism $g \in \widetilde{\mathrm{O}}\left(\Lambda_{\text {cub }}^{0},\left\langle v_{d}\right\rangle\right)$ whose class modulo $\widetilde{\mathrm{O}}\left(\Lambda_{\text {cub }}^{0}, v_{d}\right)$ generates $\mathbb{Z} / 2 \mathbb{Z}$. We will explain the construction of an explicit such $g$.

LEMMA 2.8. Let $x \in \Lambda_{\text {cub }}^{0}$ be primitive with $(x, x) \neq 0$ and $3 \chi(x, x)$. There exists an $f \in \widetilde{\mathrm{O}}\left(\Lambda_{\text {cub }}^{0}\right)$ such that $f(x)=e_{2}+f_{2} \cdot(x, x) / 2$. 
Proof. As Disc $\Lambda_{\text {cub }}^{0}=\mathbb{Z} / 3 \mathbb{Z}$, the divisibility $\operatorname{div}(x)$ of $x$ in $\Lambda_{\text {cub }}^{0}$ is either 1 or 3 . Since $3 \chi$ $(x, x)$, it must be 1 . Therefore the class of $x / \operatorname{div}(x)$ in Disc $\Lambda_{\text {cub }}^{0}$ is trivial. By Eichler's criterion [8, Proposition 3.3], there is an $f \in \widetilde{\mathrm{O}}\left(\Lambda_{\text {cub }}^{0}\right)$ sending $x$ to $e_{2}+f_{2} \cdot(x, x) / 2$.

Suppose $d \equiv 0 \bmod 6$. By Lemma $2 \cdot 8$, we can assume

$$
v_{d}=e_{2}-\frac{d}{6} f_{2} \in \Lambda_{\mathrm{cub}}=E_{8}(-1)^{\oplus 2} \oplus U_{1} \oplus U_{2} \oplus \mathbb{Z}(-1)^{\oplus 3} .
$$

Let $g \in \mathrm{O}\left(\Lambda_{\text {cub }}\right)$ be given by

$$
\left.g\right|_{E_{8}(-1)^{\oplus 2} \oplus \mathbb{Z}(-1)^{\oplus 3}}=\mathrm{id} ;\left.\quad g\right|_{U_{1} \oplus U_{2}}=-\mathrm{id} .
$$

Then we have $g\left(v_{d}\right)=-v_{d}$, so $\left.g\right|_{\Lambda_{\text {cub }}^{0}}$ generates $\widetilde{\mathrm{O}}\left(\Lambda_{\text {cub }}^{0},\left\langle v_{d}\right\rangle\right) / \widetilde{\mathrm{O}}\left(\Lambda_{\text {cub }}^{0}, v_{d}\right)$.

\section{The involution on $\mathcal{M}_{d}$}

From now on, we will assume that $d$ satisfies (**). In the previous section, we explained that the map

$$
\bar{\gamma}: \mathcal{Q D}\left(\Lambda_{d}\right) \longrightarrow \widetilde{\mathrm{O}}\left(\Lambda_{\mathrm{cub}}^{0},\left\langle v_{d}\right\rangle\right) \backslash \mathcal{D}\left(K_{d}^{\perp}\right)
$$

is an isomorphism if $d \equiv 2 \bmod 6$ and has degree two if $d \equiv 0 \bmod 6$. In the second case, define $\tau: \mathcal{Q D}\left(\Lambda_{d}\right) \rightarrow \mathcal{Q D}\left(\Lambda_{d}\right)$ to be the covering involution of $\bar{\gamma}$. It is induced by the map $\left.g\right|_{K_{d}^{\perp}}$, seen as an element of $\widetilde{\mathrm{O}}\left(\Lambda_{d}\right)$. Note that $\tau$ maps $\mathcal{M}_{d}$ to itself: As explained in e.g. [11, remark 6.3.7], the complement of $\mathcal{M}_{d}$ in $\mathcal{Q D}\left(\Lambda_{d}\right)$ is

$$
\bigcup_{\delta \in \Lambda_{d}, \delta^{2}=-2} \delta^{\perp}
$$

and this set is clearly preserved under $g$.

Proposition 3.1. The morphism $\tau$ does not depend on the choice of isomorphism $\Lambda_{d} \cong K / d$.

Proof. Precomposing the isomorphism $\Lambda_{d} \cong K_{d}^{\perp}$ with $f \in \mathrm{O}\left(\Lambda_{d}\right)$ changes $g$ on $\Lambda_{d}$ to $f^{-1} \circ g \circ f$. Note that this has the same action on the abelian group $\operatorname{Disc}\left(\Lambda_{d}\right)$ as $g$, thus it induces the same action on $\mathcal{Q D}\left(\Lambda_{d}\right)$.

For a $\mathrm{K} 3$ surface $S$, we denote by $\widetilde{\mathrm{H}}(S, \mathbb{Z})$ the full cohomology of $S$ with the Mukai pairing and the Hodge structure of weight two defined by $\widetilde{\mathrm{H}}^{2,0}(S):=\mathrm{H}^{2,0}(S)$.

Let $H$ be a generic polarisation on $S$. For a primitive vector $v=(r, \ell, s) \in \widetilde{\mathrm{H}}(S, \mathbb{Z})$, denote by $M_{S}(v)$ the moduli space of $H$-stable coherent sheaves on $S$ with Mukai vector $v$. Recall $\left[\mathbf{1 1}\right.$, Chapter 10] that if there exists a $w$ in $\widetilde{\mathrm{H}}^{1,1}(S, \mathbb{Z})=\mathrm{H}^{0}(S, \mathbb{Z}) \oplus \mathrm{H}^{1,1}(S, \mathbb{Z}) \oplus \mathrm{H}^{4}(S, \mathbb{Z})$ with $(v, w)=1$, then this is a fine moduli space. If $r>0$ (or $(\ell)^{2} \geq 2$ and $(\ell, H)>0$ ) and $(v)^{2}=0$, then $M_{S}(v)$ is a K3 surface.

THEOREM 3.2. Let $\left(S^{\tau}, L^{\tau}\right)=\tau(S, L)$. Then $S^{\tau}$ is isomorphic to the moduli space $M_{S}(v)$ of stable coherent sheaves on $S$ with Mukai vector $v=(3, L, d / 6)$. Under the natural identification $\mathrm{H}^{2}\left(S^{\tau}, \mathbb{Z}\right) \cong v^{\perp} / \mathbb{Z} v$, we have $L^{\tau}=(d,(d / 3-1) L, d / 3(d / 6-1))$. 
Let us describe the strategy of the proof. Since the map $g$ does not induce the identity on Disc $\Lambda_{d}$, it does not extend to an orthogonal transformation of $\Lambda_{\mathrm{K} 3}$. However, we will show that $g$ extends to $\widetilde{g} \in \mathrm{O}\left(\widetilde{\Lambda}_{\mathrm{K} 3}\right)$. Using $\widetilde{g}$, we find $S^{\tau}$ as follows. Let $x$ be a representative of the period of $(S, L)$ in $\Lambda_{d, \mathbb{C}} \subset \widetilde{\Lambda}_{\mathrm{K} 3, \mathbb{C}}$. The K3 surface $S^{\tau}$ is the one whose period can be represented by $g(x) \in \Lambda_{d}, \mathbb{C}$. The map $\widetilde{g}$ induces a Hodge isometry $\widetilde{\mathrm{H}}(S, \mathbb{Z}) \cong \widetilde{\mathrm{H}}\left(S^{\tau}, \mathbb{Z}\right)$, so by the derived Torelli theorem ([18], see also [11, Proposition 16.3.5]), $S$ and $S^{\tau}$ are Fourier-Mukai partners.

More precisely, denote by $\widetilde{g}_{\text {Muk }}$ the morphism $\widetilde{g}$, seen as an orthogonal transformation of $\Lambda_{\text {Muk }}$. Let $v=(r, \ell, s):=\widetilde{g}_{\text {Muk }}^{-1}(0,0,1)$. There exists a universal sheaf $\mathcal{E}$ on $S \times M_{S}(v)$. Let $\Phi_{\mathcal{E}}^{H}: \widetilde{\mathrm{H}}\left(M_{S}(v), \mathbb{Z}\right) \rightarrow \widetilde{\mathrm{H}}(S, \mathbb{Z})$ be the induced cohomological Fourier-Mukai transform, which satisfies $\Phi_{\mathcal{E}}^{H}(0,0,1)=v$. Then $\widetilde{g}_{\text {Muk }} \circ \Phi_{\mathcal{E}}^{H}$ sends $\mathrm{H}^{2}\left(M_{S}(v), \mathbb{Z}\right)$ to $\mathrm{H}^{2}\left(S^{\tau}, \mathbb{Z}\right)$, which shows that $S^{\tau}$ is isomorphic to $M_{S}(v)$.

To describe $L^{\tau}$, note that $\Phi_{\mathcal{E}}^{H}$ induces an isomorphism $\mathrm{H}^{2}\left(M_{S}(v), \mathbb{Z}\right) \cong v^{\perp} / \mathbb{Z} v$, where $v^{\perp} \subset \widetilde{\mathrm{H}}(S, \mathbb{Z})$ (this is a result by Mukai, see [11, Remark 10·3·7]). Thus, $\widetilde{g}_{\text {Muk }}^{-1}$ restricts to an isomorphism $\mathrm{H}^{2}\left(S^{\tau}, \mathbb{Z}\right) \cong v^{\perp} / \mathbb{Z} v$. Under this identification, the polarisation $L^{\tau}$ is mapped to $\widetilde{g}_{\mathrm{Muk}}^{-1}\left(\ell_{d}\right)$.

Remark 3.3. The extension $\widetilde{g}$ of $g$ is not unique. But if $\widetilde{g}^{\prime}$ is another extension, then $\tilde{g}_{\text {Muk }}^{-1} \circ \widetilde{g}_{\text {Muk }}^{\prime}$ is an orthogonal transformation of $\Lambda_{\text {Muk }}$ sending $v^{\prime}=\left(\widetilde{g}_{\text {Muk }}^{\prime}\right)^{-1}(0,0,1)$ to $v$. This induces a Hodge isometry $\mathrm{H}^{2}\left(M_{S}\left(v^{\prime}\right), \mathbb{Z}\right) \cong \mathrm{H}^{2}\left(M_{S}(v), \mathbb{Z}\right)$, so $M_{S}\left(v^{\prime}\right)$ and $M_{S}(v)$ are isomorphic.

Remark 3.4. The space $\mathcal{Q D}\left(\Lambda_{d}\right)$ can be interpreted as the moduli space of quasi-polarised $\mathrm{K} 3$ surfaces, i.e. pairs $(S, L)$ with $L$ the class of a big and nef line bundle [10, Section 5]. For such pairs the theorem is still valid.

Remark 3.5. For $d \equiv 0 \bmod 6$, the ramification locus of $\bar{\gamma}$ over $\mathcal{M}_{d}$ consists of those $(S, L)$ which are polarised isomorphic to $\left(S^{\tau}, L^{\tau}\right)$. It follows from [10, Section 8] that $\bar{\gamma}$ is unramified over $\left\{(S, L) \in \mathcal{M}_{d} \mid \rho(S)=1\right\}$.

\subsection{Proof of Theorem $3 \cdot 2$}

We first compute the action of $g$ on Disc $K_{d}^{\perp} \cong$ Disc $K_{d}$. Suppose $T \in K_{d}$ is a primitive element such that $h$ and $T$ generate $K_{d}$. Then $(h, T)$ is divisible by 3. We can write $v_{d}=$ $(h, T) h / 3$, which has square $-d / 3$. It follows that $K_{d}=\mathbb{Z} h \oplus \mathbb{Z} v_{d}$, so

$$
\operatorname{Disc} K_{d} \cong \mathbb{Z} / 3 \mathbb{Z} \oplus \mathbb{Z} /\left(\frac{d}{3}\right) \mathbb{Z},
$$

with the action of $g$ given by $i d \oplus-$ id.

LEMMA 3.6. The induced action of $g$ on $\operatorname{Disc} \Lambda_{d} \cong \mathbb{Z} / d \mathbb{Z}$ is given by $x \mapsto(d / 3-1) x$.

Proof. If we reduce modulo $d / 3$, multiplication by $d / 3-1$ equals - id. If we reduce modulo 3 , it is the identity, as $d / 3-1 \equiv 1 \bmod 3$.

To extend $g$ on $\Lambda_{d}$ to $\widetilde{\Lambda}_{\mathrm{K} 3}$ it thus suffices, by Lemma $1 \cdot 1$, to find an orthogonal transformation of $\Lambda_{d}^{\perp}=\mathbb{Z} \ell_{d} \oplus U_{4}$ acting on the discriminant group by $x \mapsto(d / 3-1) x$. Consider $u \in \mathrm{O}\left(\mathbb{Z} \ell_{d} \oplus U_{4}\right)$ defined by (see Remark 3.7) 


$$
\begin{aligned}
& e_{4} \longmapsto-\frac{d}{6} e_{4}-\frac{1}{3}\left(\frac{d}{6}-1\right) \ell_{d}+\frac{1}{3}\left(\frac{d}{6}-1\right)^{2} f_{4} \\
& f_{4} \longmapsto 3 e_{4}+\ell_{d}-\frac{d}{6} f_{4} \\
& \ell_{d} \longmapsto d e_{4}+\left(\frac{d}{3}-1\right) \ell_{d}-\frac{d}{3}\left(\frac{d}{6}-1\right) f_{4}
\end{aligned}
$$

One computes that this is an involution. The discriminant group $\operatorname{Disc}\left(\mathbb{Z} \ell_{d} \oplus U_{4}\right) \cong \mathbb{Z} / d \mathbb{Z}$ is generated by the class $\overline{\ell_{d} / d}$, which $\bar{u}$ multiplies by $d / 3-1$.

It follows that $g \oplus u \in \mathrm{O}\left(\Lambda_{d} \oplus \mathbb{Z} \ell_{d} \oplus U_{4}\right)$ extends to $\widetilde{g} \in \mathrm{O}\left(\widetilde{\Lambda}_{\mathrm{K} 3}\right)$. Since $\widetilde{g}$ is an involution, we have $\tilde{g}^{-1}\left(f_{4}\right)=\tilde{g}\left(f_{4}\right)=3 e_{4}+\ell_{d}-(d / 6) f_{4}$. As an element of the Mukai lattice, this is

$$
v=\left(3, \ell_{d}, d / 6\right) \in \Lambda_{\mathrm{Muk}}=\mathbb{Z} e_{4} \oplus \Lambda_{\mathrm{K} 3} \oplus \mathbb{Z}\left(-f_{4}\right) .
$$

The polarisation $L^{\tau}=\widetilde{g}^{-1}\left(\ell_{d}\right)$, seen as an element of $v^{\perp} / \mathbb{Z} v \subset \widetilde{\mathrm{H}}(S, \mathbb{Z})$, is

$$
L^{\tau}=\left(d,\left(\frac{d}{3}-1\right) L, \frac{d}{3}\left(\frac{d}{6}-1\right)\right) .
$$

This completes the proof of Theorem $3 \cdot 2$.

Remark 3.7. The map $u$ can be found as follows. One extends $\left.g\right|_{K_{d}^{\perp}}$ to $\widetilde{\Lambda}_{\mathrm{K} 3}$ by taking id $\oplus-$ id on the orthogonal complement $A_{2}(-1) \oplus \mathbb{Z}(d / 3)$ of $K_{d}^{\perp}$ in $\widetilde{\Lambda}_{\mathrm{K} 3}$. In order to find the Mukai vector $v$, one writes down an explicit isomorphism $A_{2}(-1) \oplus \mathbb{Z}(d / 3) \cong \mathbb{Z} \ell_{d}^{\prime} \oplus U_{4}$ and computes the corresponding orthogonal transformation $u$ of $\mathbb{Z} \ell_{d}^{\prime} \oplus U_{4}$. We chose not to spell out these tedious computations.

Remark 3.8. As shown in the proof of Proposition 3·1, we can replace $g$ by any element of $\mathrm{O}\left(\Lambda_{d}\right)$ with the same action on Disc $\Lambda_{d}$. For instance, we can take the automorphism given by the identity on $E_{8}(-1)^{\oplus 2} \oplus U_{1}$ and $u$ on $U_{2} \oplus \mathbb{Z}\left(e_{3}-(d / 2) f_{3}\right) \cong\left(\mathbb{Z} \ell_{d} \oplus U_{4}\right)(-1)$. This allows us to define $\tau$ on $\mathcal{M}_{d}$ for all $d \equiv 0 \bmod 6$ with $d / 6 \equiv 1 \bmod 3$.

\section{Birationality of Hilbert schemes}

In this section we study the Hilbert schemes of $n$ points $\operatorname{Hilb}^{n}(S)$ and $\operatorname{Hilb}^{n}\left(S^{\tau}\right)$ of our K3 surfaces $S$ and $S^{\tau}$. Corollary 4.9 and the results in Section 4.2 hold for all $d$ such that $d / 6 \equiv 1 \bmod 3$, using Remark 3.8.

\subsection{Hilbert schemes of two points}

For a cubic fourfold $X$ we denote by $F(X)$ the Fano variety of lines on $X$, a fourdimensional hyperkähler variety of $\mathrm{K} 3^{[2]}$ type. Hassett proved the following:

THEOREM 4.1 ([9, Theorem 6·1.4]). Assume that d satisfies

$$
d=2\left(n^{2}+n+1\right)
$$

for some integer $n \geq 2$. Let $X$ be a generic cubic fourfold in $\mathcal{C}_{d}$. Then $F(X)$ is isomorphic to $\operatorname{Hilb}^{2}(S)$, where $(S, L) \in \mathcal{M}_{d}$ is associated to $X$. 
If also $d \equiv 0$ mod 6, then $F(X)$ is isomorphic to both $\operatorname{Hilb}^{2}(S)$ and $\operatorname{Hilb}^{2}\left(S^{\tau}\right)$ (Hassett calls $F(X)$ ambiguous). Since birationality specialises in families of hyperkähler manifolds, it follows that $\operatorname{Hilb}^{2}(S)$ is birational to $\mathrm{Hilb}^{2}\left(S^{\tau}\right)$ for all K3 surfaces $S$ of degree $d$. We can generalise this using the following result by Addington. See also Remark 4.6.

THEOREM 4.2 ([1, Theorem 2]). A cubic fourfold X lies in $\mathcal{C}_{d}$ for some d satisfying

$$
a^{2} d=2\left(n^{2}+n+1\right)
$$

if and only if $F(X)$ is birational to $\mathrm{Hilb}^{2}(S)$ for some $K 3$ surface $S$.

Note that $(* * *)$ implies $(* *)$.

Lemma 4.3. Suppose that $d$ satisfies (***). Then there exists a choice of the rational map $\varphi: \mathcal{M}_{d} \rightarrow \mathcal{C}_{d}$ such that if $(S, L) \in \mathcal{M}_{d}$ is associated to $X \in \mathcal{C}_{d}$ via $\varphi$, then $\operatorname{Hilb}^{2}(S)$ and $F(X)$ are birational.

Proof. Consider the sublattices

$$
K_{d}^{\perp} \oplus T \subset \tilde{\Lambda}_{\mathrm{K} 3} \supset \Lambda_{d} \oplus \mathbb{Z} \ell_{d} \oplus U_{4},
$$

where $T \supset A_{2}=\left\langle\lambda_{1}, \lambda_{2}\right\rangle$ is the orthogonal complement of $K / d$ in $\widetilde{\Lambda}_{\mathrm{K} 3}$. Then $d$ satisfies (**) if and only if $T \cong \mathbb{Z} \ell_{d} \oplus U_{4}$. Addington showed that (***) holds if and only if $\psi: T \rightarrow$ $\mathbb{Z} \ell_{d} \oplus U_{4}$ can be chosen such that $\psi\left(\lambda_{1}\right)=e_{4}+f_{4}$. Extend $\psi$ to an element of $\mathrm{O}\left(\widetilde{\Lambda}_{\mathrm{K} 3}\right)$ (use Lemma $1 \cdot 1$ and [11, Theorem 14.2.4]) and let $\varphi$ be the induced map $\mathcal{M}_{d} \rightarrow \mathcal{C}_{d}$.

Assume that $(S, L) \in \mathcal{M}_{d}$ is associated to $X \in \mathcal{C}_{d}$ via $\varphi$. Choose an isomorphism $\mathrm{H}^{2}(S, \mathbb{Z}) \cong U_{4}^{\perp} \subset \widetilde{\Lambda}_{\mathrm{K} 3}$ sending $L$ to $\ell_{d}$, and consider the induced Hodge structure on $\widetilde{\Lambda}_{\mathrm{K} 3}$. There are isometries of sub-Hodge structures

$$
\mathrm{H}^{2}(F(X), \mathbb{Z}) \cong \lambda_{1}^{\perp} \cong \psi\left(\lambda_{1}\right)^{\perp}=\left(e_{4}+f_{4}\right)^{\perp} \cong \mathrm{H}^{2}\left(M_{S}(1,0,-1), \mathbb{Z}\right),
$$

where the sign in $M_{S}(1,0,-1)$ appears because we view the Mukai vector as an element of $\Lambda_{\text {Muk }}$. By Markman's birational Torelli theorem for manifolds of K3 ${ }^{[n]}$ type [15, Corollary 9.9], $F(X)$ is birational to $M_{S}(1,0,-1) \cong \operatorname{Hilb}^{2}(S)$.

COROLlary 4.4. When $d \equiv 0$ mod 6 satisfies $(* * *)$, then $\operatorname{Hilb}^{2}(S) \sim_{\text {bir }} \operatorname{Hilb}^{2}\left(S^{\tau}\right)$ for any $K 3$ surface $(S, L) \in \mathcal{M}_{d}$.

The following proposition shows that we have more than just birationality: if $d$ is such that $\operatorname{Hilb}^{2}(S) \sim$ bir $\operatorname{Hilb}^{2}\left(S^{\tau}\right)$, then for $S$ generic, $\operatorname{Hilb}^{2}(S)$ and $\operatorname{Hilb}^{2}\left(S^{\tau}\right)$ are isomorphic.

Proposition 4.5. Let $(S, L)$ be a polarised $K 3$ surface of degree $d$ with $\operatorname{Pic}(S)=\mathbb{Z} L$ and $3 \mid d$. Then $\mathrm{Hilb}^{2}(S)$ has only one birational model.

Proof. By [6, Theorem 5.1] the walls of the ample cone of $\operatorname{Hilb}^{2}(S)$ in the interior of the movable cone are given by the hypersurfaces $x^{\perp} \subset \mathrm{NS}\left(\operatorname{Hilb}^{2}(S)\right) \otimes \mathbb{R}$ for all $x \in \operatorname{NS}\left(\operatorname{Hilb}^{2}(S)\right)$ of square -10 and divisibility two. We will show that there are no such $x$.

There is an isomorphism

$$
\mathrm{NS}\left(\operatorname{Hilb}^{2}(S)\right) \cong \mathrm{NS}(S) \oplus \mathbb{Z} \delta=\mathbb{Z} L \oplus \mathbb{Z} \delta,
$$


where $\delta$ is a (-2)-class orthogonal to $L$ [5]. So any class in $\mathrm{NS}^{2}\left(\operatorname{Hilb}^{2}(S)\right)$ is given by $a L+$ $b \delta$ for some $a, b \in \mathbb{Z}$, and its square is $a^{2} d-2 b^{2}$. Setting this equal to -10 gives the Pell equation $b^{2}-a^{2} d / 2=5$ which, after reducing modulo 3 , gives $b^{2} \equiv 2 \bmod 3$. This is not possible.

It follows that under any birational map $\operatorname{Hilb}^{2}(S) \rightarrow Y$ the pullback of an ample class is ample, thus the map is an isomorphism [7].

Remark 4.6. This also implies that when $d$ satisfies $(* * *)$ and $3 \mid d$, then for a generic cubic fourfold $X$ of discriminant $d, F(X)$ is actually isomorphic to $\operatorname{Hilb}^{2}(S)$ for a $\mathrm{K} 3$ surface $S$ associated to $X$. When $3 \chi d$, Proposition 4.5 does not hold. For example, consider $d=38$, which satisfies $(* * *)$ but not the condition in Theorem $4 \cdot 1$. The element $2 L+9 \delta$ has square -10 and divisibility $\operatorname{gcd}(2,18)=2$. Hence, $\operatorname{Hilb}^{2}(S)$ has more than one birational model.

It is natural to ask for the exact conditions on $d$ for $\operatorname{Hilb}^{2}(S)$ to be birational to $\operatorname{Hilb}^{2}\left(S^{\tau}\right)$, for all $S$ of degree $d$. It turns out that (***) is too strong. We use the following two results by [16].

PRoposition $4 \cdot 7$ ([16, Proposition 2.1]). Let $(S, L)$ be a polarised K3 surface satisfying $\operatorname{Pic}(S)=\mathbb{Z} L$. Let $v=(x, c L, y)$ be a primitive isotropic Mukai vector such that $M_{S}(v)$ is a fine moduli space. Then $v=\left(p^{2} r, p q L, q^{2} s\right)$ for some integers $p, r, q, s$ with $\operatorname{gcd}(p r, q s)=$ 1 and $(L)^{2}=2 r s$, and there is an isomorphism $M_{S}(v) \cong M_{S}(r, L, s)$. Moreover, $M_{S}(r, L, s)$ is isomorphic to $M_{S}\left(r^{\prime}, L, s^{\prime}\right)$ if and only if $\{r, s\}=\left\{r^{\prime}, s^{\prime}\right\}$.

Proposition 4.8 ([16, Theorem 2.2]). Let $S_{1}$ and $S_{2}$ be derived equivalent $K 3$ surfaces of Picard number one. Then $\operatorname{Hilb}^{n}\left(S_{1}\right)$ and $\operatorname{Hilb}^{n}\left(S_{2}\right)$ are birational if and only if $S_{2}$ is isomorphic to $M_{S_{1}}\left(p^{2} r, p q L, q^{2} s\right)$ for some $p, q$ with $p^{2} r(n-1)-q^{2} s= \pm 1$. Moreover, $\{r, s\}$ is uniquely determined by $S_{2}$.

Note that $p^{2} r(n-1)-q^{2} s= \pm 1$ is equivalent to $\left((1,0,1-n),\left(p^{2} r, p q L, q^{2} s\right)\right)= \pm 1$. So when $\left(p^{2} r, p q L, q^{2} s\right)$ is primitive then $M_{S_{1}}\left(p^{2} r, p q L, q^{2} s\right)$ is a fine moduli space, isomorphic (also when $\rho\left(S_{1}\right)>1$ ) to $M_{S_{1}}(r, L, s)$ by Proposition 4.7.

Our description of $\tau$ gave us $S^{\tau}=M_{S}(3, L, d / 6)$, so $r=3$ and $s=d / 6$. Thus, Proposition 4.8 tells us that when $\rho(S)=1$, then we have $\operatorname{Hilb}^{n}(S) \sim_{\text {bir }} \operatorname{Hilb}^{n}\left(S^{\tau}\right)$ if and only if there exist non-zero integers $p, q$ such that $3 p^{2}(n-1)-(d / 6) q^{2}= \pm 1$. Note that $3 p^{2}(n-1)-(d / 6) q^{2}=1$ does not happen in our case: since $d / 6 \equiv 1 \bmod 3$, reducing modulo 3 gives $q^{2} \equiv 2$ mod 3 which is not possible.

COROLlary 4.9. Suppose that $\rho(S)=1$. Then $\operatorname{Hilb}^{2}(S)$ and $\operatorname{Hilb}^{2}\left(S^{\tau}\right)$ are birational if and only if there exists an integral solution to the equation

$$
3 p^{2}-(d / 6) q^{2}=-1 .
$$

Equivalently, $\mathrm{Hilb}^{2}(S)$ admits a line bundle of degree 6.

Proof. A class $a L+b \delta$ in NS(Hilb $\left.{ }^{2}(S)\right)=\mathbb{Z} L \oplus \mathbb{Z} \delta$ has square $a^{2} d-2 b^{2}=6$, in particular $b=3 b_{0}$ for some $b_{0}$, if and only if $3 b_{0}^{2}-(d / 6) a^{2}=-1$.

Note that when $\rho(S)>1$, the existence of a solution to (4.1) still implies that $\operatorname{Hilb}^{2}(S)$ and $\operatorname{Hilb}^{2}\left(S^{\tau}\right)$ are birational and that $\operatorname{Hilb}^{2}(S)$ admits a line bundle of degree 6 . 
Table I. Conditions $(* *),(* * *)$ and (4-1) for small values of $d$

\begin{tabular}{|c|c|c|c|c|c|c|c|c|c|}
\hline$d / 6$ & $d$ & $(* *)$ & $(* * *)$ & $(4 \cdot 1)$ & $d / 6$ & $d$ & $(* *)$ & $(* * *)$ & $(4 \cdot 1)$ \\
\hline 1 & 6 & $\mathrm{x}$ & $\mathrm{x}$ & $x$ & 52 & 312 & & & \\
\hline 4 & 24 & & & $\mathrm{x}$ & 55 & 330 & & & \\
\hline 7 & 42 & $\mathrm{x}$ & $\mathrm{x}$ & $\mathrm{x}$ & 58 & 348 & & & \\
\hline 10 & 60 & & & & 61 & 366 & $\mathrm{x}$ & $\mathrm{x}$ & $\mathrm{x}$ \\
\hline 13 & 78 & $\mathrm{x}$ & & $\mathrm{x}$ & 64 & 384 & & & \\
\hline 16 & 96 & & & & 67 & 402 & $\mathrm{x}$ & $\mathrm{x}$ & $\mathrm{x}$ \\
\hline 19 & 114 & $\mathrm{x}$ & $\mathrm{x}$ & $\mathrm{x}$ & 70 & 420 & & & \\
\hline 22 & 132 & & & & 73 & 438 & $\mathrm{x}$ & & \\
\hline 25 & 150 & & & & 76 & 456 & & & $\mathrm{x}$ \\
\hline 28 & 168 & & & $\mathrm{x}$ & 79 & 474 & $\mathrm{x}$ & $\mathrm{x}$ & $\mathrm{x}$ \\
\hline 31 & 186 & $\mathrm{x}$ & $\mathrm{x}$ & $\mathrm{x}$ & 82 & 492 & & & \\
\hline 34 & 204 & & & & 85 & 510 & & & \\
\hline 37 & 222 & $\mathrm{x}$ & $\mathrm{x}$ & $\mathrm{x}$ & 88 & 528 & & & \\
\hline 40 & 240 & & & & 91 & 546 & $\mathrm{x}$ & $\mathrm{x}$ & $\mathrm{x}$ \\
\hline 43 & 258 & $\mathrm{x}$ & $\mathrm{x}$ & $\mathrm{x}$ & 94 & 564 & & & \\
\hline 46 & 276 & & & & 97 & 582 & $\mathrm{x}$ & & \\
\hline 49 & 294 & $\mathrm{x}$ & & $\mathrm{x}$ & 100 & 600 & & & \\
\hline
\end{tabular}

Condition (***) implies that (4.1) is solvable. Namely, assume we have $a^{2} d / 2=n^{2}+$ $n+1$. Multiplying with 4 gives $(2 a)^{2} d / 2=(2 n+1)^{2}+3$. As $d$ is divisible by 3 , so is $2 n+1$, and we find that $3((2 n+1) / 3)^{2}-(2 a)^{2} d / 6=-1$.

In fact, (***) is equivalent to the existence of a solution to (4.1) with $p$ odd and $q$ even. One can show that such a solution always exists when $d / 6$ is a prime $m \equiv 3 \bmod 4$. The following example shows that there exist $d$ for which (4.1) is solvable but (***) does not hold.

Example 4.10. Let $d=78$, which satisfies $(* *)$ but not (***) (see [1]). Equation (4.1) holds with $p=2$ and $q=1$. It follows that $\operatorname{Hilb}^{2}(S) \sim_{\text {bir }} \operatorname{Hilb}^{2}\left(S^{\tau}\right)$ for any $S$ of degree 78. Moreover, when $\rho(S)=1$, Proposition 4.5 tells us that $\operatorname{Hilb}^{2}(S)$ and $\operatorname{Hilb}^{2}\left(S^{\tau}\right)$ are isomorphic (even though $S$ and $S^{\tau}$ are not).

Next, we give an example where (4.1) is not solvable. As $S$ and $S^{\tau}$ are derived equivalent, so are $\operatorname{Hilb}^{n}(S)$ and $\operatorname{Hilb}^{n}\left(S^{\tau}\right)$ for all $n \geq 1$ [19, Proposition. 8]. Therefore, we obtain two derived equivalent Hilbert schemes of two points on $\mathrm{K} 3$ surfaces which are not birational. The first example of this phenomenon was given in [16, Example 2.5]. Note the similarity between $S^{\tau}$ and the K3 surface $Y$ in [16, Proposition 1.2].

Example 4.11. Consider $d=6 \cdot 73$. This again satisfies $(* *)$ but not $(* * *)$. Note that $(4 \cdot 1)$ holds if and only if $(3 p)^{2}-(d / 2) q^{2}=-3$, which is equivalent to $x^{2}-(d / 2) y^{2}=-3$ when 3 divides $d$. This is a usual Pell type equation and one can easily check (using e.g. [3, theorem 4.2.7]) that it has no solution for $d=6 \cdot 73$. So for $S$ generic of degree $6 \cdot 73$, $\operatorname{Hilb}^{2}(S)$ is not birational to $\operatorname{Hilb}^{2}\left(S^{\tau}\right)$.

In Table I we give an overview of which $d \leq 600$ with $d / 6 \equiv 1$ mod 3 satisfy the numerical conditions $(* *),(* * *)$ and $(4 \cdot 1)$ (see also [1]). 


\subsection{Higher-dimensional Hilbert schemes}

We have seen that $\operatorname{Hilb}^{n}(S)$ and $\operatorname{Hilb}^{n}\left(S^{\tau}\right)$ are birational if and only if there is a solution to

$$
3 p^{2}(n-1)-(d / 6) q^{2}=-1 .
$$

We give some examples for low $n$.

$n=3$. The lowest $d$ satisfying (**) and $6 \mid d$ is $d=42$. Equation (4.2) with $n=3$ reads $6 p^{2}-7 q^{2}=-1$, which is solved by $p=q=1$. In general, one can show that if $d / 6$ is a prime $m \equiv 5,7 \bmod 8$, then $\operatorname{Hilb}^{3}(S) \sim_{\text {bir }} \operatorname{Hilb}^{3}\left(S^{\tau}\right)$.

$n=4$. In this case, equation $(4 \cdot 2)$ reads $(3 p)^{2}-(d / 6) q^{2}=-1$. This is always solvable when $d / 6$ is a prime $m \equiv 1 \bmod 4$. Namely, note that when $m>2$ is prime, then $x^{2}-m y^{2}=$ -1 has a solution if and only if $m \equiv 1 \bmod 4$. Reducing this modulo 3 gives $x^{2}-y^{2} \equiv$ $-1 \bmod 3$. This implies that $x \equiv 0 \bmod 3$. Writing $x=3 x^{\prime}$ gives $9\left(x^{\prime}\right)^{2}-(d / 6) y^{2}=-1$, i.e. (4.2) with $n=4$.

$n=5$. Equation $(4 \cdot 2)$ is given by $3(2 p)^{2}-(d / 6) q^{2}=-1$, which is a solution for $(4 \cdot 1)$. This shows that $\operatorname{Hilb}^{5}(S) \sim_{\text {bir }} \operatorname{Hilb}^{5}\left(S^{\tau}\right)$ implies $\operatorname{Hilb}^{2}(S) \sim_{\text {bir }} \operatorname{Hilb}^{2}\left(S^{\tau}\right)$.

Acknowledgements. This work was part of my research as a $\mathrm{PhD}$ candidate. I am grateful to my advisor Daniel Huybrechts for many helpful suggestions and comments. I would also like to thank Giovanni Mongardi and Andrey Soldatenkov for their help, and Pablo Magni for comments on the first version. Finally, I want to thank the referee for useful comments.

This work was supported by the Bonn International Graduate School of Mathematics and the SFB/TR 45 'Periods, Moduli Spaces and Arithmetic of Algebraic Varieties' of the DFG (German Research Foundation).

\section{REFERENCES}

[1] N. Addington. On two rationality conjectures for cubic fourfolds. Math. Res. Lett. 23 (2016), 1-13.

[2] N. AdDington and R. Thomas. Hodge theory and derived categories of cubic fourfolds. Duke Math. J. 163 (2014), 1885-1927.

[3] T. Andreescu, D. Andrica and I. Cucurezeanu. An introduction to Diophantine equations. (Birkhäuser Verlag, New York, 2010).

[4] W. L. BAILY and A. Borel. Compactification of arithmetic quotients of bounded symmetric domains. Ann. of Math. 84 (1966), 442-528.

[5] A. Beauville. Variétés Kähleriennes dont la première classe de Chern est nulle. J. Differential Geom. 18 (1983), 755-782.

[6] O. DeBARRE and E. MACRÌ. On the period map for polarised hyperkähler fourfolds. Int. Math. Res. Not. IMRN. No. 22 (2019), 6887-6923.

[7] A. FUJIKI. A theorem on bimeromorphic maps of Kähler manifolds and its applications. Publ. Res. Inst. Math. Sci. textbf17 (1981), 735-754.

[8] V. Gritsenko, K. Hulek and G. Sankaran. Abelianisation of orthogonal groups and the fundamental group of modular varieties. J. Algebra 322 (2009), 463-478.

[9] B. HassetT. Special cubic fourfolds. Compositio Math. 120 (2000), 1-23.

[10] K. HuleK and D. Ploog. Fourier-Mukai partners and polarised K3 surfaces. In Arithmetic and geometry of K3 surfaces and Calabi-Yau threefolds, Fields Inst. Commun. vol. 67 (Springer, New York, 2013), pp. 333-365.

[11] D. HuYBREChTS. Lectures on K3 surfaces. Camb. Stud. Adv. Maths. no. 158 (Cambridge University Press, 2016).

[12] D. Huybrechts. The K3 category of a cubic fourfold. Compositio Math. 153 (2017), 586-620.

[13] R. LAZA. The moduli space of cubic fourfolds via the period map. Ann. of Math. (2) 172 (2010), 673-711.

[14] E. LoolJEnga. The period map for cubic fourfolds. Invent. Math. 177 (2009), 213-233. 
[15] E. MARKMAN. A survey of Torelli and monodromy results for holomorphic-symplectic varieties. In Complex and Differential Geometry, Springer Proc. Math. vol. 8 (Springer, Berlin, Heidelberg, 2011), pp. 257-322.

[16] C. Meachan, G. Mongardi and K. Yoshioka. Derived equivalent Hilbert schemes of points on K3 surfaces which are not birational. Math. Z. (2019), to appear

[17] K. Oguiso. K3 surfaces via almost-primes. Math. Res. Lett. 9 (2002). 47-63.

[18] D. O. OrLov. Equivalences of derived categories and K3 surfaces. J. Math. Sci. 84 (1997), 13611381.

[19] D. Ploog. Equivariant autoequivalences for finite group actions. Adv. Math. 216 (2007), 62-74.

[20] I. SaTA KE. Algebraic Structures of Symmetric Domains (Princeton University Press, Princeton, 1980).

[21] Y. Zarhin. Algebraic cycles over cubic fourfolds. Boll. Un. Mat. Ital. B (7) 4 (1990), 833-847.

[22] S. ZuCKER. The Hodge conjecture for cubic fourfolds. Compositio Math. 34 (1977), 199-209. 